\title{
DISTRIBUTED COMPRESSED SENSING OF HYPERSPECTRAL IMAGES VIA BLIND SOURCE SEPARATION
}

\author{
Mohammad Golbabaee, Simon Arberet and Pierre Vandergheynst \\ Signal Processing Institute, Ecole Polytechnique Fédérale de Lausanne (EPFL), Switzerland \\ E-mail:\{mohammad.golbabaei, simon.arberet, pierre.vandergheynst\}@epfl.ch
}

\begin{abstract}
This paper describes a novel framework for compressive sampling (CS) of multichannel signals that are highly dependent across the channels. In this work, we assume few number of sources are generating the multichannel observations based on a linear mixture model. Moreover, sources are assumed to have sparse/compressible representations in some orthonormal basis. The main contribution of this paper lies in 1) rephrasing the CS acquisition of multichannel data as a compressive blind source separation problem, and 2) proposing an optimization problem and a recovery algorithm to estimate both the sources and the mixing matrix (and thus the whole data) from the compressed measurements. A number of experiments on the acquisition of Hyperspectral images show that our proposed algorithm obtains a reconstruction error between $10 \mathrm{~dB}$ and $15 \mathrm{~dB}$ less than other state-of-the-art CS methods.
\end{abstract}

Index Terms - Compressed sensing, Blind source separation, Hyperspectral images, Mixture model, Sparse approximation, Dictionary learning.

\section{INTRODUCTION}

Hyperspectral images (HSI) are a collection of hundreds of images which have been acquired simultaneously in narrow and adjacent spectral bands, and finds many applications including agriculture, mineral exploration and environmental monitoring $[1,2]$.

As it is costly to acquire each pixel of the HSI, it becomes very interesting to use the compressive sampling $(\mathrm{CS})$ approach $[3,4]$ to acquire HSI. If a data $x \in \mathbb{R}^{N}$ has a sparse or compressible representation in some basis, CS is an alternative to the Shannon/Nyquist sampling, which reduces the number $M$ of measurements required to acquire the data $x$ i.e., $M<N$. In practice, the CS measurements can be done using the single-pixel hyperspectral camera (SPHC) [5].

We assume like in $[1,2]$ that the HSI data $X \in \mathbb{R}_{+}^{J \times N}$, wherein $J$ is the number of the spectral channels and $N$ is the resolution (number of pixels) of the image at each channel of the HSI, is generated from a linear source mixture model $X=A S$, where the matrix $A \in \mathbb{R}_{+}^{J \times I}$ is called the mixing matrix and the matrix $S \in \mathbb{R}_{+}^{I \times N}$ is called the source matrix.

The main contribution of this paper is to exploit this mixture model as an underlying structure [6] so as to recover HSI with very few CS measurements. As opposed to our previous work [7], we assume that the mixture parameters $A$ are unknown and we develop an algorithm to blindly learn this matrix $A$ as well as the sources $S$ from the CS measurements. We refer to this problem as the compressive

This research was supported by Swiss National Science Foundation through grant 200021-117884 and the SMALL project funded by the EU FP7 FET-Open program. blind source separation problem, which shares certain similarities with the dictionary learning problem [8]. It mainly consists in estimating $A$ and $S$ simultaneously through an optimization problem which is not convex. Nonetheless, its objective function becomes convex if either $A$ or $S$ is fixed. Thus, our proposed algorithm follows an iterative scheme composed of the two steps below:

1. a sparse approximation step, where the sources $S$ are estimated from the CS measurements while $A$ is fixed;

2. a dictionary update step, where the mixing matrix (also called dictionary) $A$ is estimated from the CS measurements while the sources $S$ are fixed.

\section{PROBLEM SETUP}

In this section we present our linear mixing model of HSI, which will be later exploited to acquire any HSI with very few CS measurements, via a new joint decoding scheme.

\subsection{Observations Model}

Each row $X^{j}$ of the matrix $X$ (in this note, by superscript we index a row of a matrix) corresponds to a slice of the global cubic HSI i.e, a 2-D image observed in a certain spectral band (we reshape this slice into an $N$ dimensional vector). Typically there is a high dependency between the slice images of HSI (rows of $X$ ), since the whole scene to be monitored is composed of few subregions containing certain different materials. We refer to these regions as different sources. More precisely, we define a source image $S^{i} \in[0,1]^{1 \times N}$ as a positive valued vector which represents the percentage of a given material (indexed by $i$ ) in each pixel of the scene. As a consequence, for a given pixel of the scene (indexed by $n$ ), the sum of the consisting sources must be equal to one i.e., $\forall n \in\{1, \ldots, N\}$ the source images must satisfy $\sum_{i} S^{i}(n)=1$. In practice, if the spatial resolution of the image is high compared to the structural content of the image, each pixel corresponds to only one material, which means that the sources are disjoint and take their values in the set $\{0,1\}^{1 \times N}$. It also implies that the sources are orthogonal which is an assumption will be exploited later in this paper. For each material there is also a positive valued vector $A_{i} \in \mathbb{R}_{+}^{J \times 1}$ that represents the spectral reflectance, i.e. the energy of material $i$ on the different frequency channels. With descriptions above, any hyperspectral image can be decomposed by several distinct sources as following:

$$
X=A S,
$$

where the rows of $S \in\{0,1\}^{I \times N}$ and the columns of $A \in \mathbb{R}_{+}^{J \times I}$ are respectively the $I$ source images and their corresponding spectral vectors. 


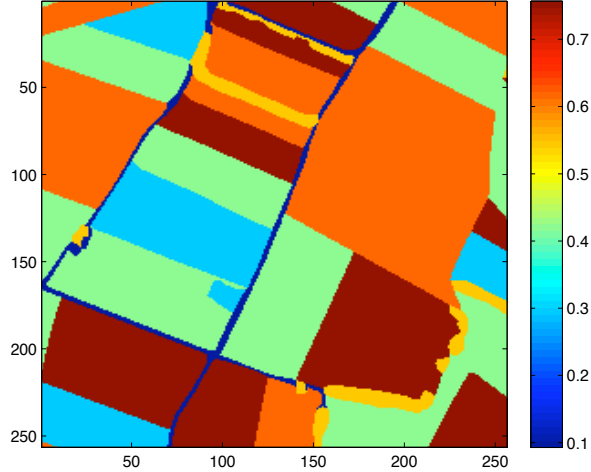

(a) Original data for channel $j=144$.

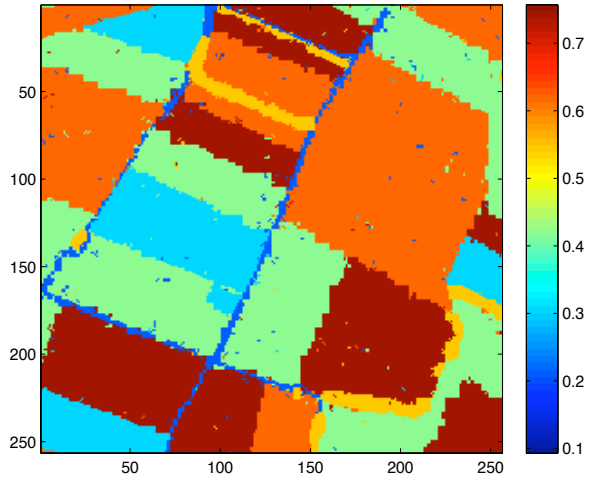

(b) Reconstruction by setting $M=3000$.

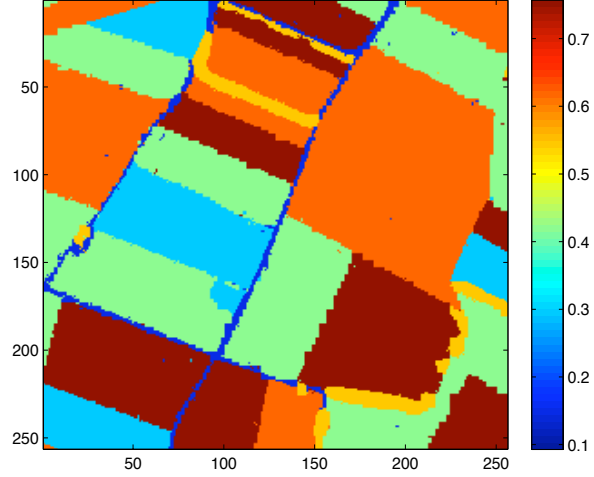

(c) Reconstruction by setting $M=4000$.

Fig. 1. Reconstruction of HSI using BSS-IHT, by randomly sampling b) $4.6 \%$ and c) $6.1 \%$ of the original cube, demonstrated for a slice/channel $j=144$.

In real applications, the mixtures $X^{j}$ and the sources $S^{i}$ have sparse/compressible representations in wavelet domain. We define matrix $\Sigma$ to be the 2D wavelet coefficients of the rows of $S$ such that $S=\Sigma \Psi^{T}$ where $\Psi$ is the $2 \mathrm{D}$ wavelet basis. Thus, from the model (1) we can deduce

$$
X=A \Sigma \Psi^{T}
$$

\subsection{Sampling Mechanism}

Each spectral channel $j$ is sampled by $M \ll N$ linear measurements $\left(Y^{j}\right)^{T}=\Phi\left(X^{j}\right)^{T}$, where $\Phi \in \mathbb{R}^{M \times N}$ is the sampling matrix which is the same for all channels. We choose it random Gaussian due to the nice properties of the random matrices for compression [4]. Stacking these samples together and taking into account the noise due to the sensors, the quantization and the eventual transmission, we form the $J \times M$ matrix of measurements:

$$
Y=X \Phi^{T}+Z
$$

where $Z \in \mathbb{R}^{J \times M}$ is the noise matrix that is assumed to have elements with i.i.d. zero mean Gaussian distribution $\mathcal{N}\left(0, \sigma^{2}\right)$.

\section{JOINT RECOVERY APPROACH}

In this section we describe our approach to recover the multichannel data $X$ from a set of compressive measurements $Y$.

\subsection{Definition of the optimization problem}

The matrices $A$ and $S$ have both positive values $(A, S \geq 0)$, and $S$ has a sparse representation $\Sigma$ in the wavelet basis $\Psi$. Also, as mentioned in section 2.1 the source image coefficients represent the percentage of a given material in a certain pixel $n$ of the scene, which implies $\sum_{i} S^{i}(n)=1$. We also assume that the sources are orthogonal because the support of the sources is mostly disjoint. This constraint results in orthogonality of their wavelet coefficients, which can be expressed by Off $\operatorname{diag}\left(\Sigma \Sigma^{T}\right)=0$, where the operator Off $\operatorname{diag}($.$) defines a zero-diagonal matrix by zeroing the diagonal$ components of its argument. The optimization problem we aim to solve can be written as:

$$
\begin{array}{cl}
\underset{A, \Sigma}{\operatorname{minimize}} & \left\|Y-A \Sigma \Psi^{T} \Phi^{T}\right\|_{F} \\
\text { subject to } & \left\|\Sigma^{i}\right\|_{0} \leq K_{i}, \quad 1 \leq i \leq I, \\
& \text { Off } \operatorname{diag}\left(\Sigma \Sigma^{T}\right)=0, \\
& A, S \geq 0, \\
& \sum_{i} S^{i}(n)=1, \quad 1 \leq n \leq N,
\end{array}
$$

with $S=\Sigma \Psi^{T}$. Note that $\|\cdot\|_{F}$ denotes the Frobenius norm of a matrix, and $\|\cdot\|_{0}\left(l_{0}\right.$-norm) counts the number of the nonzero elements in a vector (thus, $K_{i}$ is the sparsity level of the source $i$ in the wavelet domain). As we can see, (4) is not decouplable into the rows/channels, which implies a joint recovery approach

\subsection{BSS-IHT Algorithm}

As previously mentioned, the main feature of our scheme lies in recovering the sources $S$ and the mixing matrix $A$ by alternating between a sparse approximation step and a dictionary update step i.e., blind source separation (BSS) from the compressive measurements. The sparse approximation step consists in estimating $S$ with a fixed $\widehat{A}$ ( $\widehat{A}$ being the current estimate of $A$ ) and is implemented via an Iterative Hard Thresholding (IHT) [9] where each of the constraints of (4) are imposed at each iteration just after the gradient descent step. The dictionary update step consists in estimating $A$ with a fixed $\widehat{S}$ ( $\widehat{S}$ being the estimate of $S$ obtained from the previous step), and is obtained by multiplying the pseudo-inverse of $\widehat{S} \Phi^{T}$ to the right side of $Y$, and then setting the non-positive values to zero so as to impose the non-negativity constraint on $A$.

\section{EXPERIMENTS}

In this section we provide results from two sets of experiments, that demonstrate the performance of our scheme in noiseless settings. Our simulations are based on HSI synthesized using (1), where $I=6$ sources are extracted from a ground truth map image ${ }^{1}$ of farms at the suburb of Geneva city and the source spectra (i.e. matrix $A$ ) are choosen at random form the USGS digital spectral library

\footnotetext{
${ }^{1}$ We acknowledge Xavier Gigandet for providing this ground truth map.
} 


\begin{tabular}{l|c|c|c|}
\multicolumn{1}{c}{} & \multicolumn{1}{c}{ SNR of $\widehat{A}$} & \multicolumn{1}{c}{ SNR of $\widehat{S}$} & \multicolumn{1}{c}{ SNR of $\widehat{X}$} \\
\cline { 2 - 4 }$M=3000$ & $23.22 \mathrm{~dB}$ & $10.73 \mathrm{~dB}$ & $19.49 \mathrm{~dB}$ \\
\cline { 2 - 4 }$M=4000$ & $29.35 \mathrm{~dB}$ & $14.00 \mathrm{~dB}$ & $22.65 \mathrm{~dB}$ \\
\cline { 2 - 4 } & &
\end{tabular}

Table 1. Performance of BSS-IHT for recovering the mixing matrix $\widehat{A}$, the source images $\widehat{S}$, and the whole HSI cube $\widehat{X}$ of the first experiment, for two sampling regimes $\mathrm{M}=3000$ and $M=4000$.
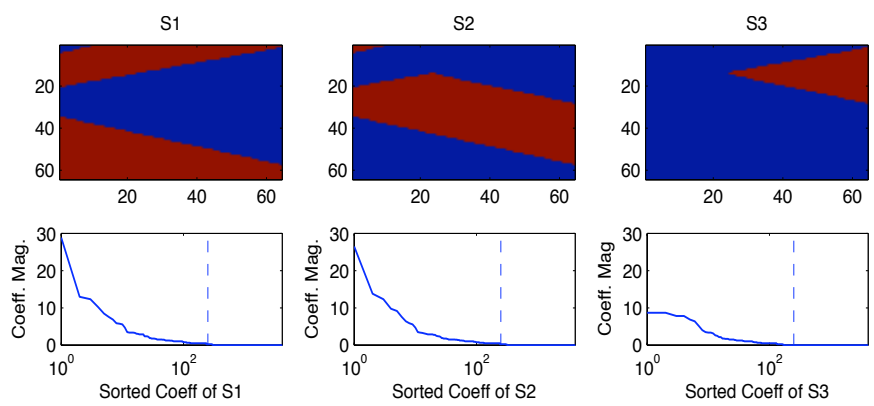

Fig. 2. Three source images of HSI from the second experiment (the three top figures, wherein red and blue pixels correspond to one and zero values respectively) and the sorted magnitude of their corresponding wavelet coefficients (the three plots below). As we can see, the energy is mainly concentrated in the $K_{i}=250$ largest wavelet coefficients of each source, indicated by the dashed line.

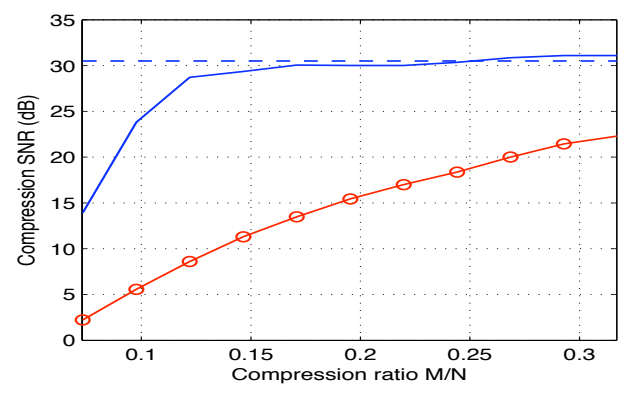

Fig. 3. Reconstruction SNR of the compressive sampled HSI for different compression ratio M/N, using BSS-ITH (blue curve) and JS-ITH (red curve) recovery methods. The dashed blue line indicates reconstruction SNR for adaptive compression scheme that keeps the $K_{i}=250$ largest wavelet coefficients of each source.

2 . Figure 1(a) shows one channel of the resulting HSI. The HSI cube consists of dimension $256 \times 256 \times 128$, which indicates slices of the resolution $N=256 \times 256$ that are taken over $J=128$ frequency bands.

Following our sampling scheme, in the first experiment, we set $M=3000$ and 4000 , to randomly sample/measure the whole HSI cube by about $4.6 \%$ and $6.1 \%$ of its original size, respectively. For recovery, we use our iterative algorithm based on estimating the 2000 strongest wavelet coefficients of each source i.e., $K_{i} \leq 2000$. Figure 1 shows one slice of the recovered HSI using our algorithm for both sampling rates. More details on recovery performance of BSS-IHT for this experiment are provided in the table 1.

In the second setup, we compare the performance of our algorithm with Iterative Hard Thresholding method using Joint-Sparsity assumption (JS-IHT). Using the same sampling scheme as in 2.2, the later method attempts to recover multichannel signals that are sparse

\footnotetext{
${ }^{2}$ Available at the url http://speclab.cr.usgs.gov/spectral.lib06.
}

and also sharing a strong common support set (nonzero coefficients are nearly at the same positions, across the channels [10]). Indeed, this assumption applies well to the hyperspectral images, since the main structures in the images are preserved across the spectral channels ( just some regions highlight more or less in different frequency ranges) that leads to a joint-sparse wavelet representation.

We choose the first $64 \times 64$ pixels of the upper left part of the images from the first experiment, and across the first 64 spectral channels, which enables us to run quick experiments that evaluates the average performance of both recovery schemes for various compression matrices of different sizes. Figure 2 shows the three existing sources of this part and with their sorted wavelet coefficients. Figure 3 demonstrates the reconstruction SNR, for both recovery schemes and for different compression sizes. The plots are averaged over 20 independent realizations of the random measurement matrix.

By knowing a priori the positions and the values of the 250 largest wavelet coefficients of each source $\left(K_{i} \leq 250\right)$, together with the knowledge of the mixing matrix $A$ (the spectral reflectance), one could reconstruct the whole HSI with SNR of $30.05 \mathrm{~dB}$ (the blue dashed line in Figure 3). Whereas, using our non-adaptive sampling/reconstrution scheme, we achieve the same reconstruction SNR, by taking only $18 \%$ samples of the whole HSI cube. At the same compression rate, comparing our recovery scheme with JSIHT, indicates more than $15 \mathrm{~dB}$ of improvement in performance.

\section{CONCLUSION}

In this paper we develop a new method for recovering sparse multichannel signals from their distributed compressive samples. Unlike the other methods for multichannel CS, our scheme attempts to improve recovery by exploiting dependencies across the channels via assuming a linear source mixture model for the signals. Our scheme recovers the whole signals, by approximating their underlying sparse sources and their mixing parameters from those few random measurements. Several experiments on the Hyperspectral images indicates a massive improvement in recovery with respect to the stateof-the-art multichannel CS methods, in particular, under very low sampling rate regimes.

\section{REFERENCES}

[1] J.C Harsanyi and C.-I Chang, "Hyperspectral image classification and dimensionality reduction: an orthogonal subspace projection approach," Geoscience and Remote Sensing, IEEE Transactions on DOI - 10.1109/36.298007, vol. 32, no. 4, pp. 779-785, 1994.

[2] Jing Wang and Chein-I Chang, "Independent component analysis-based dimensionality reduction with applications in hyperspectral image analysis," Geoscience and Remote Sensing, IEEE Transactions on DOI - 10.1109/TGRS.2005.863297, vol. 44, no. 6, pp. 1586- 1600, 2006.

[3] E.J. Candes, "The restricted isometry property and its implications for compressed sensing," Comptes rendus-Mathématique, vol. 346, no. 9-10, pp. 589-592, 2008.

[4] D.L. Donoho, "Compressed sensing," IEEE Transactions on Information Theory, vol. 52 , no. 4, pp. 1289-1306, 2006.

[5] M.F. Duarte, M.A. Davenport, D. Takhar, J.N. Laska, T. Sun, K.F. Kelly, and R.G. Baraniuk, "Single-pixel imaging via compressive sampling," IEEE Signal Processing Magazine, vol. 25, no. 2, pp. 83-91, 2008.

[6] R.G Baraniuk, V Cevher, MF Duarte, and C Hegde, "Model-based compressive sensing," preprint, 2008

[7] M. Golbabaee, S. Arberet, and P. Vandergheynst, "Multichannel compressed sensing via source separation for hyperspectral images," in Eusipco, 2010.

[8] M Aharon, M Elad, and A Bruckstein, "K-svd: An algorithm for designing overcomplete dictionaries for sparse representation," Signal Processing, IEEE Transactions on DOI - 10.1109/TSP.2006.881199, vol. 54, no. 11, pp. 4311-4322, 2006.

[9] T Blumensath and M Davies, "Iterative thresholding for sparse approximations," Journal of Fourier Analysis and Applications, Jan 2008.

[10] M.F. Duarte, S. Sarvotham, D. Baron, M.B. Wakin, and R.G. Baraniuk, "Distributed compressed sensing of jointly sparse signals," in Asilomar Conf. Signals, Sys., Comput, 2005, pp. 1537-1541. 